\title{
Asean Harmonization on Traditional Medicines
}

\author{
Suwijiyo Pramono* \\ Faculty of Pharmacy, Universitas Gadjah Mada Yogyakarta, Indonesia \\ *Corresponding author: Suwijiyo Pramono, Faculty of Pharmacy, Universitas Gadjah Mada Yogyakarta, Indonesia. \\ To Cite This Article: Suwijiyo Pramono. Asean Harmonization on Traditional Medicines. Am J Biomed Sci \& Res. 2019-4(2). AJBSR.MS.ID.000776. \\ DOI: 10.34297/AJBSR.2019.04.000776
}

Received: July 02, 2019 | Published: July 23, 2019

\section{Definition of Traditional Medicines}

Term "Traditional Medicines" has two meanings. The first one means all healing methods that are used traditionally by the people as curative, preventive, rehabilitative, paliative and promotive manner for their health. It can be psychological, physical and/or herbal interventions. An exemple of psychological intervention is supranatural approach, while accupuncture and accupressure are the exemples of physical interventions. The third intervention is the use of herbal based on empirical or traditional knowledge of the people so it is also mentioned as traditional medicine. This article is focused on the second meaning of traditional medicine i.e the use of herbal medicine based on empirical or traditional knowledge. In point view of free trade market, ten ASEAN member states have created harmonization on traditional medicines. Several guidelines have been established and two of these are close related to biomedical aspect of traditional medicines i.e Guidelines on Safety substantiation and Guidelines on Claims and Claim substantiations [1].

The aims of harmonization are to harmonize and implement the technical requirements and guidelines for traditional medicines trade so to reduce technical barriers to trade among ASEAN Member States and contributing to the ASEAN Economic integration initiative without compromising the safety, quality and efficacy/claimed benefits of products. According to the ASEAN Agreements the definition of traditional medicine is any product for human use consisting of active ingredients derived from natural sources (plants, animals and/or minerals) used in the system of traditional practice. It shall not include any sterile preparation, vaccines, any substance derived from human parts, any isolated and characterized chemical substances.

\section{Guidelines of Safety Substantiation}

In the guidelines of safety substantiation is mentioned that safety data requirements for traditional medicines are required to substantiate the safety profile of products to be marketed and to assure the safety of product in the market. Safety substantiation might not be required for traditional medicine products containing ingredients with documented well established safety profile, such as traditional medicines with a long history of safe use. Safety data might be required for the following, but not limited to:

a) New ingredients

b) Ingredients derived from new methods of purification or extraction/ manufacturing

c) Ingredients/products derived from the existing one with new combination, new dosage, new delivery system and use in special or new target population (e.g. pregnant, lactating women, children, etc). Existing ingredients/products with safety concern. Safety concern may be newly emerging or established, and in some cases may need additional information to support safe usage in traditional medicine.

d) Safety data, including toxicity data, could be derived from animal and/or human studies using internationally accepted methodologies including acute, sub-chronic and/or chronic toxicity data may be required. Other toxicity data such as teratogenicity, carcinogenicity, and/or mutagenicity data may be required, when necessary.

\section{Guideline on Claims and Claims Substantiation}

The ASEAN Guidelines on Claims and Claims Substantiation for Traditional Medicines is developed by taking into consideration similar guidelines that exist internationally (WHO, EU, US, Canada, Australia) and the regulatory situation and stakeholders'interests in the ASEAN region. The traditional medicine claims refer to any message that states, suggests, or implies that a traditional medicine ingredient/product has positive contribution and benefit to human health. A balanced approach between consumer protection and encouraging science and innovation is important in implementing the harmonised ASEAN Guidelines on Claims and Claims Substantiation for Traditional Medicines so the Guideline aims to provide guidance on making unbiased and truthful claims, supported by adequate evidence in order to protect the consumers from misleading claims. This will enable consumers to make informed choices in taking care of their health. Furthermore, the document will facilitate the product placement of traditional 
medicine products and set up requirements for efficacy data submission for certain traditional medicine claims. Data supporting safety and claim/efficacy of product is the evidence of documented traditional use or history of use that may be found in the following: Classical Traditional Medicine Text, Pharmacopoeias and Monographs, Refferences/Textbooks or Report of Etnomedicine Survey.

\section{References}

1. (2016) ASEAN Traditional Medicines Scientific Committee (ATSC), Guidelines on Safety substantiation and Guidelines on Claims and Claim substantiations. 